\title{
Simulation of the radiation fallout from gamma-ray measurements
}

\author{
Anatoli A. Gusev ${ }^{1}$ Inacio M. Martin ${ }^{1,2} \cdot$ Mauro A. Alves ${ }^{2}$ Alessandro J. de Abreu
}

Received: 28 January 2015/ Accepted: 23 June 2015/Published online: 25 July 2015

(C) Springer International Publishing Switzerland 2015

\begin{abstract}
A method of a simulation of radiation fallout concomitant atmospheric liquid precipitations is proposed. The fallout is reconstructed from observed gammas through a numerical solution of the first kind Volterra integral equation describing variation of nuclide amount produced by precipitating ${ }^{214} \mathrm{~Pb}$ and ${ }^{214} \mathrm{Bi}$. Application of the method is considered on an example of rainfalls in Brazil. There were observed short fallout enhancements in the initial phases of the rains. It was demonstrated that nuclide quantity per unit of precipitated water decreases with increasing rainfall rate. The results obtained are compared with other observations and models.
\end{abstract}

Keywords Gamma radiation - Rainfall - Radon gas · Radio nuclide's in raindrops

\section{Introduction}

Radon and its progenies are natural radioactive nuclides found in the atmosphere all over the globe. Studies of the natural atmospheric radiation are of a great importance in applications as human health safety and radiation security of nuclear plants. Observation of various component of the natural and anthropogenic radiation is a helpful instrument in studies of atmospheric chemistry and physics of aerosols, cloud formation and atmospheric mass transport.

Alessandro J. de Abreu

abreu.alessandro@gmail.com

1 Space Research Institute of the Russian Academy of Sciences, Moscow, Russia

2 Instituto Tecnológico de Aeronáutica, ITA, Divisão de Ciências Fundamentais, São José dos Campos, SP, Brazil
${ }^{222} \mathrm{Rn}$ and ${ }^{220} \mathrm{Rn}$ radon isotopes are produced in decay chains of primordial ${ }^{238} \mathrm{U}$ and ${ }^{232} \mathrm{Th}$ contained in the earth's crust. Basing on cosmological notions the ${ }^{232} \mathrm{Th} /{ }^{238} \mathrm{U}$ abundance ratio is estimated at $2-4$. However, because of their half-life ratio $\tau_{232 \mathrm{Th}} / \tau_{238 \mathrm{U}}=14.1 \times 10^{9} /$ $4.5 \times 10^{9}$ year $=3.13$ the production rate of radon isotopes should be quite close. The local ${ }^{222} \mathrm{Rn} /{ }^{220} \mathrm{Rn}$ production ratio depends on geological characteristics of the terrain, but their near-ground concentrations are generally similar in order of magnitude.

After exalting from the earth, radon being an inert gas is able easily disperses into the atmosphere not adhering by itself to the precipitants. However ${ }^{220} \mathrm{Rn}$ due to relatively short half-life $\tau_{220 \mathrm{Ra}}=55 \mathrm{~s}$ remains quite near to the ground providing noticeable ${ }^{208} \mathrm{Tl}$ gamma line in the decay chain:

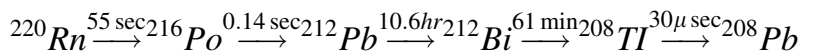

(the values above the arrows indicate half-life of the corresponding radionuclide).

Opposite to that ${ }^{222} \mathrm{Rn}$ with its much longer half-life $\tau_{222 \mathrm{Ra}}=3.8$ day disperse into the whole atmosphere populating it with progenies from the decay chain:

${ }^{222} \mathrm{Rn} \stackrel{3.8 \text { day }_{2}}{\longrightarrow}{ }^{218} \mathrm{Po} \stackrel{3.1 \mathrm{~min}_{2}}{\longrightarrow}{ }^{14} \mathrm{~Pb} \stackrel{26.8 \min _{2}}{\longrightarrow}{ }^{214} \mathrm{Bi} \stackrel{20 \min _{2}}{\longrightarrow}{ }^{14} \mathrm{Po} \stackrel{164 \mu \sec _{2}}{\longrightarrow}{ }^{210} \mathrm{~Pb}$

Owing to their longer half-life ${ }^{214} \mathrm{~Pb}$ and ${ }^{214} \mathrm{Bi}$ are the most abundant radon progenies in the atmosphere. The solid radon progenies become airborne and immediately attach to the dust particles, aerosols and water droplets existing in the atmosphere. These particles undergo intensive convection aligning its concentration in the atmosphere (Kumar et al. 1999). The droplets in clouds 
aggregate into raindrop and precipitate to the ground surface. The concentration of radionuclides in raindrops determines specific radionuclide fallout characterized by radioactivity per unit of liquid precipitations.

All the decays are accompanied by emission of $\gamma$-quanta carrying away the excess potential energy of the daughter nucleus. The energy of the emitted $\gamma$-quantum depends on the decaying nuclide. Owning to that it is possible to detect the presence of a specific radionuclide in the environment through detection and measuring energy quanta emitted.

In spite of the fact that basic physics of cloud formation and precipitation is known (Nobuyoshi and Katase 1982) no comprehensive quantitative model of the phenomenon has still been developed, due to the insufficient accuracy of knowledge of parameters of the processes in clouds. From this point of view, the observation of the fallout dynamics may be a helpful instrument for verification of current models and adjusting their parameters. The half-lives of ${ }^{214} \mathrm{~Pb}$ and ${ }^{214} \mathrm{Bi}$ are on the same scale as precipitation events, that allows reliable correlation of atmospheric and radiation observation.

Radiochemical methods and solid state track detectors allowing direct identification of radionuclides are rather complicated that hampers their usage for continues longterm observations. Opposite to that measurement of fallout $\gamma$-emission can be performed with relatively simple scintillation (e.g. Paatero and Hatakka 1999) and solid-state detectors (e.g. Ichiji and Hattori 2000). The results of numerical simulations (Nishikawa et al. 1989) demonstrate that during a rainfall a crystal or solid-state detector registers at the ground level gammas emitted from the encircling surface. A corresponding radius increases with the energy of $\gamma$-quanta from $80 \mathrm{~m}$ for $0.2 \mathrm{MeV}$ to $200 \mathrm{~m}$ for $3 \mathrm{MeV}$. A certain difficulty of these measurements is rather low fallout intensity in comparison with the background. Due to that, the measurements require large detector volumes and/or accumulation of the rainfall water to increase concentration of precipitated radionuclide in the immediate vicinity of the detector. It is especially important if not only overall gamma intensity is measured but its energy distribution.

Using this experimental approach Horng and Jiang (2004) evaluated a raindrop growth time in a cloud, Greenfield et al. (2008) estimated the time of raindrops passage from a cloud to the ground. Greenfield et al. (2003) also explored a possibility of monitoring rainfall rate through $\gamma$-ray observation. In all this studies rainfall sampling interval was not shorter than a quarter of an hour.

Brazil is ranked second in natural radioactivity in the world and first in that among populated area (UNSCEAR 2000). For example the average values of the radium equivalent activities were evaluated in Meaipe (ES) as $83425 \mathrm{~Bq} \mathrm{~kg}^{-1}$ (Veiga et al. 2006) that is 225 times above the limit of $370 \mathrm{~Bq} \mathrm{~kg}^{-1}$ recommended for the safe use of building materials for dwellings by OECD (1979). Due to that monitoring of primordial radionuclides and its progenies is of special interest that country (e.g. Evangelista et al. 2012; Cardoso et al. 2011; Santos et al. 2008).

City of São José dos Campos is one of the most important industrial and research centers in Latin America with emphasis in aerospace science and technology. A laboratory of environmental radiation of Centro Técnico

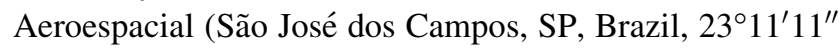
$\mathrm{S}, 45^{\circ} 52^{\prime} 43^{\prime \prime} \mathrm{W}, 650 \mathrm{~m}$ altitude) implements a continuous monitoring of near ground radiation of natural and anthropogenic origin both with the aim of pollution control and for studies in the area of atmospheric physics. The station facilities allow registration of both neutral (gammas, neutrons) and charged particles. They include several scintillation detectors of various volumes, gas-discharge detectors and $\alpha$-particles detectors. This paper presents recent results of observation of radiation dynamics related with rainfalls in the region (Table 1).
Table 1 Characteristics of the rainfalls July 23-25 2013

\begin{tabular}{llll}
\hline Parameter & Units & Rain A & Rain B \\
\hline Location on timescale of Figs. 4 and 5 & $\mathrm{min}$ & $1800-3600$ & $3600-5000$ \\
Duration & $\mathrm{h}$ & 25 & 22 \\
Precipitation level & $\mathrm{mm}$ & 8 & 60 \\
Mean rainfall & $\mathrm{mm} / \mathrm{h}$ & 0.32 & 3 \\
Maximal rainfall rate & $\mathrm{mm} / \mathrm{h}$ & 2 & 30 \\
Mean raindrop diameter & $\mathrm{mm}$ & 0.7 & 0.8 \\
Total fallout & $\mathrm{mq}$ & 50 & 60 \\
Mean $\gamma$-rate & $\mathrm{Bq} / \mathrm{min}$ & 2 & 3 \\
Maximal of $\gamma$-rate enhancement & $\mathrm{Bq} / \mathrm{min}$ & 4000 & 8000 \\
Mean specific fallout & $\mathrm{Bq} / \mathrm{mm}$ & 5.1 & 0.9 \\
\hline
\end{tabular}

a The mean raindrop diameter is calculated from the Marshall and Palmer (1948) empirical relationship $N_{\text {rd }}(d)=8000 \exp \left(R^{-0.21} d\right)$, where $d$ is a raindrop diameter and $R$ is f rain rate in $\mathrm{mm} / \mathrm{h}$ confirmed by later measurements (Peters et al. 2001) of Doppler shift 
Table 2 Number and equation presented in the text

\begin{tabular}{|c|c|}
\hline Number & Equation \\
\hline 3 & $\begin{array}{l}N_{214 \mathrm{~Pb}}(T)=F_{214 \mathrm{~Pb}}(t) e^{-\frac{T-t}{t_{214 \mathrm{~Pb}}}} \quad(\mathrm{a}) \\
N_{214 \mathrm{Bi}}(T)=F_{214 \mathrm{Bi}}(t) e^{-\frac{T-t}{t_{214 \mathrm{Bi}}}}+F_{214 \mathrm{~Pb}}(t) t_{214 \mathrm{Bi}} \frac{e^{-\frac{T-t}{t_{2144 b}}}-e^{-\frac{T-t}{t_{1244 \mathrm{~B}}}}}{t_{214 \mathrm{~Pb}}-t_{214 \mathrm{Bi}}} \quad(\mathrm{b})\end{array}$ \\
\hline 4 & $G(T)=N_{214 \mathrm{~Pb}}(T) / \tau_{214 \mathrm{~Pb}}+2 N_{214 \mathrm{Bi}}(T) / \tau_{214 \mathrm{Bi}}$ \\
\hline 5 & $G(T)=\int_{0}^{T}\left\{\frac{1}{\tau_{214 \mathrm{~Pb}}} F_{214 \mathrm{~Pb}}(t) e^{\frac{T-t}{\tau_{214 \mathrm{~Pb}}}}+\frac{2}{\tau_{214 \mathrm{Bi}}} F_{214 \mathrm{Bi}}(t) e^{\frac{T-t}{\tau_{214 \mathrm{Bi}}}}+2 F_{214 \mathrm{~Pb}}(t) \frac{\tau_{214 \mathrm{~Pb}}}{\tau_{214 \mathrm{Bi}}} \frac{\frac{T-t}{\tau^{2} 214 \mathrm{~Pb}}-e^{\frac{T-t}{\tau_{2144 \mathrm{Bi}}}}}{\tau_{214 \mathrm{~Pb}}-\tau_{214 \mathrm{Bi}}}\right\} d t$ \\
\hline 6 & $G(T)=\int_{0}^{T} F(t) K(T-t) d t$ \\
\hline 7 & $K(T-t)=\frac{1}{t_{214 \mathrm{~Pb}}} e^{-\frac{T-t}{t_{214 \mathrm{~Pb}}}}+\frac{2 e}{t_{214 \mathrm{Bi}}} e^{-\frac{T-t}{t_{214 \mathrm{Bi}}}}+2 \frac{t_{214 \mathrm{~Pb}}}{t_{214 \mathrm{Bi}}} \frac{e^{-\frac{T-t}{214 \mathrm{~Pb}}}-e^{-\frac{T-t}{2 t_{14 \mathrm{Bi}}}}}{t_{214 \mathrm{~Pb}}-t_{214 \mathrm{Bi}}}$ \\
\hline 8 & $\mathrm{~F}(i h)=\frac{1}{K(0)}\left\{2\left(\frac{(G(i h)-G(i-1) h)}{h}-\sum_{j=1}^{i=1} F(j h) K((i-j+1) h)-K((i-j) h)\right)-K(h)\right\}$ \\
\hline
\end{tabular}

\section{Method and experiment}

A fallout i.e. an amount $F(t)_{214 \mathrm{~Pb} / 214 \mathrm{Bi}}$ of radionuclides ${ }^{214} \mathrm{~Pb}$ and ${ }^{214} \mathrm{Bi}$ precipitated in a moment $t$ results due to decay over time $T$ in a radionuclide amount $N(T)_{214 \mathrm{~Pb} / 214 \mathrm{Bi}}$ as: see Eq. 3 of the Table 2 .

The second term in the right-hand part of the Eq. (3b) describes an input of ${ }^{214} \mathrm{Bi}$ radionuclides produced in the decay of the fallen ${ }^{214} \mathrm{~Pb}$. (see details in Horng and Jiang 2004). The resulting radionuclide amount is $N(T)=N_{214 \mathrm{~Pb}}(T)+N_{214 \mathrm{Bi}}(T)$.

The interim ${ }^{214} \mathrm{Po}$ (see Eq. 2) isotope is not included to the system because it practically instantaneously $\left(\tau_{214 \mathrm{Po}}=164 \mu \mathrm{s}\right)$ decays to the stable ${ }^{210} \mathrm{~Pb}$ and its input to the overall $\gamma$-flux is negligible.

The corresponding instantaneous $\gamma$-intensity $G(T)$ is: see Eq. 4 of the Table 2.

A factor 2 in $N_{214 \mathrm{Bi}}$ amount accounts for the ${ }^{214} \mathrm{Po}$ decay.

In the absence of a direct ${ }^{214} \mathrm{Bi}$ fallout $\left(F_{214 \mathrm{Bi}}(t)=0\right)$ and $(T-t) \rightarrow \infty$ the solution of Eq. 3 of the Table 2 represents so called transient equilibrium with the ratio $N_{214 \mathrm{~Pb}}(t) / N_{214 \mathrm{Bi}}(t)=\varepsilon_{\mathrm{d}}=\tau_{214 \mathrm{Bi}} /\left(\tau_{214 \mathrm{~Pb}}-\right.$ $\left.\tau_{214 \mathrm{Bi}}\right)=2.74$. In the case of time independent fallout rate $F_{214 \mathrm{~Pb}}(t)=$ const, $F_{214 \mathrm{Bi}}(t)=0$ and $T \rightarrow \infty$ the ratio $N_{214 \mathrm{Bi}}(t) / N_{214 \mathrm{~Pb}}(t)=\varepsilon_{\mathrm{e}}=\tau_{214 \mathrm{Bi}} / \tau_{214 \mathrm{~Pb}}=0.74$ describes a so called secular equilibrium, when the rates of the isotope production in the decay chain are equal and their concentrations are constant. The corresponding ratio of $\gamma$-ray rates is $G_{214 \mathrm{Bi}}(t) / G_{214 \mathrm{~Pb}}(t)=1$. In the real atmosphere the $N_{214 \mathrm{Bi}} / N_{214 \mathrm{~Pb}}$ ratio can differ from $\varepsilon_{\mathrm{e}}$ (see for example Forkapić et al. 2013): Integration of Eq. 3 of the Table 2 over $t$ results to: see Eq. 5 of the Table 2.

When the ratio $F_{214 \mathrm{Bi}}(t) / F_{214 \mathrm{~Pb}}(t)=\varepsilon_{\mathrm{c}}$ is known/assumed and does not depend on time, Eq. 3 of the Table 2 transforms to a classic linear first kind Volterra integral equation with a difference kernel $K(T-t)$
(Polyanin and Manzhirov 2008): see Eq. 6 of the Table 2.

In our case an unknown function $F(t)=\varepsilon F_{214 \mathrm{~Pb}}(t)$ and: see Eq. 7 of the Table 2.

Equation 5 of the Table 2 has a unique solution and can be solved in quadratures (Linz 1985). Equation 8 describes a numerical step by step procedure specially developed for our specific case, where $h$ is the step value nad $i h=T$ and $j h=t$.

In comparison with general algorithms of numerical solution of this type equation, it allows to avoid a straightforward solving of high order systems of linear equations which in our case exceeds one hundred. Due to that it is simpler in usage than universal codes (e.g. Press et al. 1992) and is easy adaptable for popular software [e.g. "Mathematica" (http://www.wolfram.com/mathematica)]. As a matter of fact the Eq. 8 can be reduced to the standard algorithm but is more evident and understandable. Correctness of the equation was checked with an inverse procedure i.e. simulation of $N(T)$ in Eq. 3 with reconstructed fallout $F(t)$ as an input (see below).

The gamma total $\gamma$-ray output $G$ and spectrum were measured in the $0.03-10 \mathrm{MeV}$ energy range with two commercially available $\gamma$-ray detector Ludium 44-20 with $3^{\prime \prime} \times 3^{\prime \prime} \mathrm{NaI}(\mathrm{Tl})$ scintillation crystals optically coupled with photomultiplier tube (PMT).

\section{Results}

A measured gamma spectrum in Fig. 1 demonstrates a presence of ${ }^{222} \mathrm{Ra}$ progenies: ${ }^{214} \mathrm{~Pb}(0.295 \mathrm{MeV}$ peak $),{ }^{214} \mathrm{Bi}$ $\left(0.609,1.052 \mathrm{MeV}\right.$ peaks) and that of ${ }^{220} \mathrm{Rn}:{ }^{208} \mathrm{Tl}$ (2.6 MeV peak). The strongest $1.46 \mathrm{MeV}$ line is produced by primordial ${ }^{40} \mathrm{~K}$ radionuclide.

Figure 2 presents an example of a $\gamma$-rate record for a March 19-April 232012 in the end of a rainy season 
Fig. 1 A gamma spectrum measured with $3^{\prime \prime} \times 3^{\prime \prime} \mathrm{NaI}(\mathrm{Tl})$ scintillation detector near ground level in the region

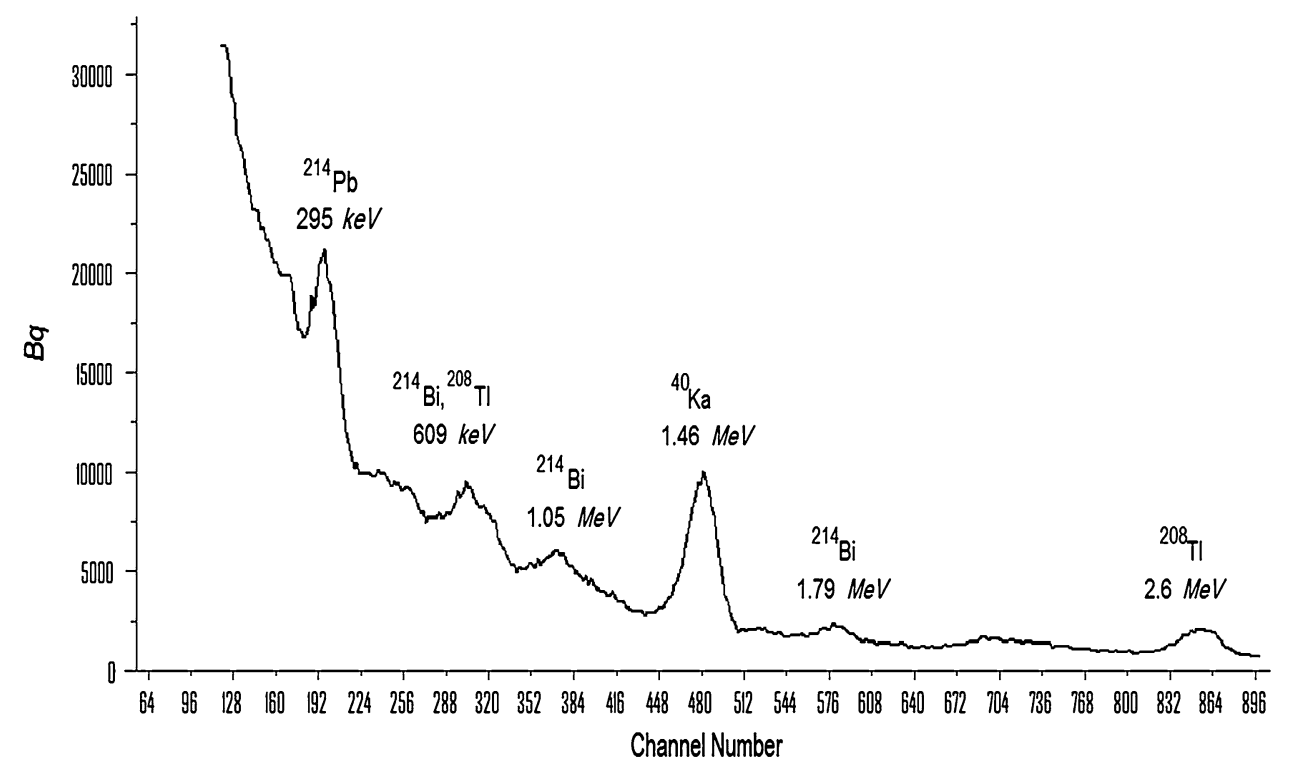

Fig. 2 Time history of the $\gamma$-ray detector. Abbreviations in grey bold marks events of precipitation: $F$ fog, $R W S$ weak short rain, $R L$ shower with lightning, $R D$ lasting drizzling rain. Permanent background component of $40,0001 / \mathrm{s}$ is subtracted

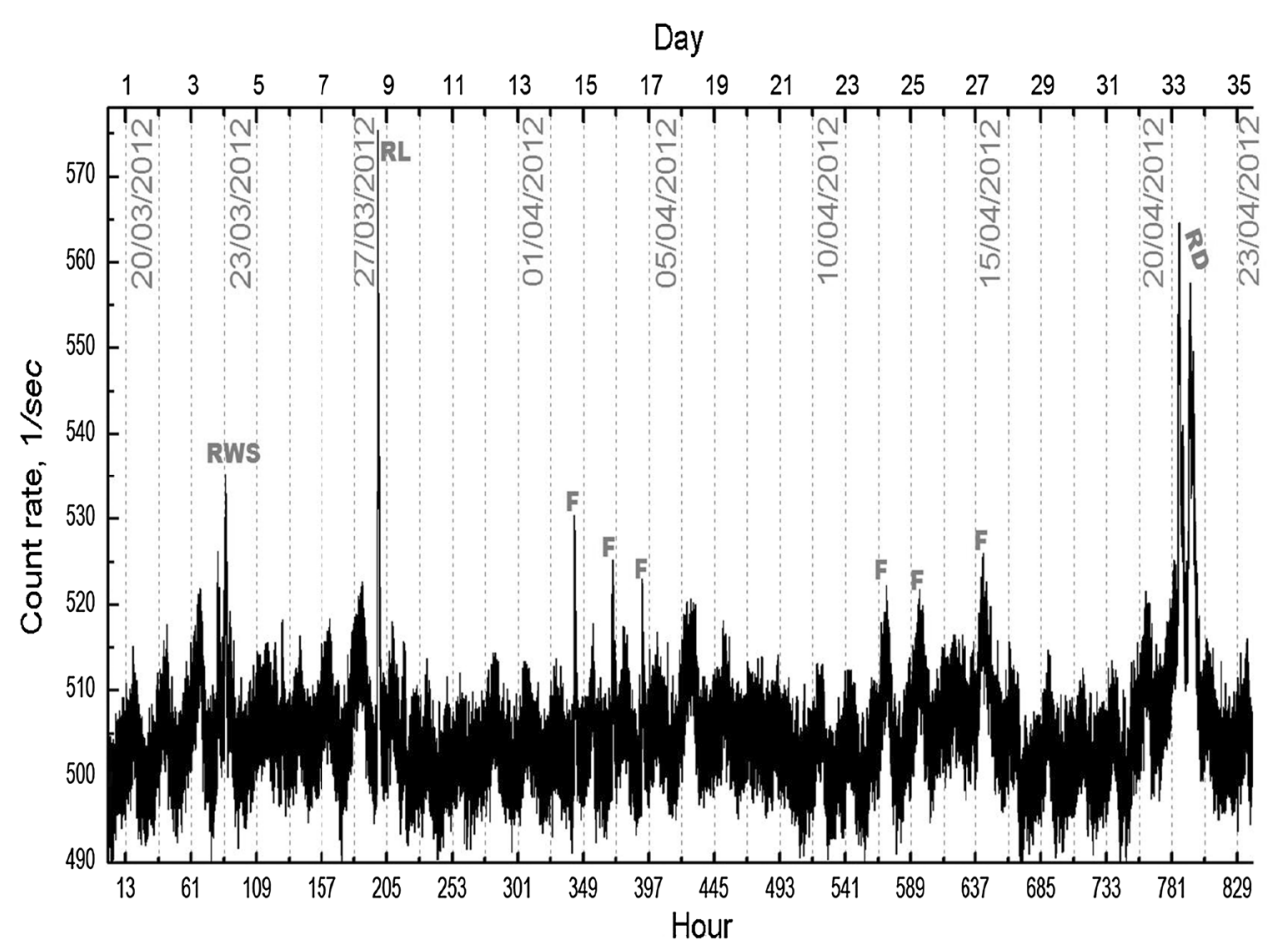

characterized with a quite low level of liquid precipitation. Clear diurnal oscillation of the $\gamma$-ray intensity of about $5 \%$ is controlled by small scale eddy dynamics of the low atmospheric boundary layer due to variations of the temperature and humidity of the atmosphere and soil.

One can see that all the variations with amplitude exceeding $\approx 520 \mathrm{~Bq} / \mathrm{s}$ are accompanied with rains or fogs (the latter also can be considered as some type of precipitation). During the rainfalls $\gamma$-ray intensity increases up to $15 \%$.
Several examples of these events are depicted in Fig. 3 in an enlarged scale. The shortest leading edge of observed $\gamma$-ray enhancements is about $10 \mathrm{~min}$. Thus corresponding variations of the fallout and rainfall rate are of the same or shorter time. Due to that, routine precipitation data of $1 \mathrm{~h}$ or lower resolution provided by meteorological stations are not adequate for such studies. To provide necessary data a set of instruments for simultaneous observation of the natural gamma background and meteorological parameters with time up to 

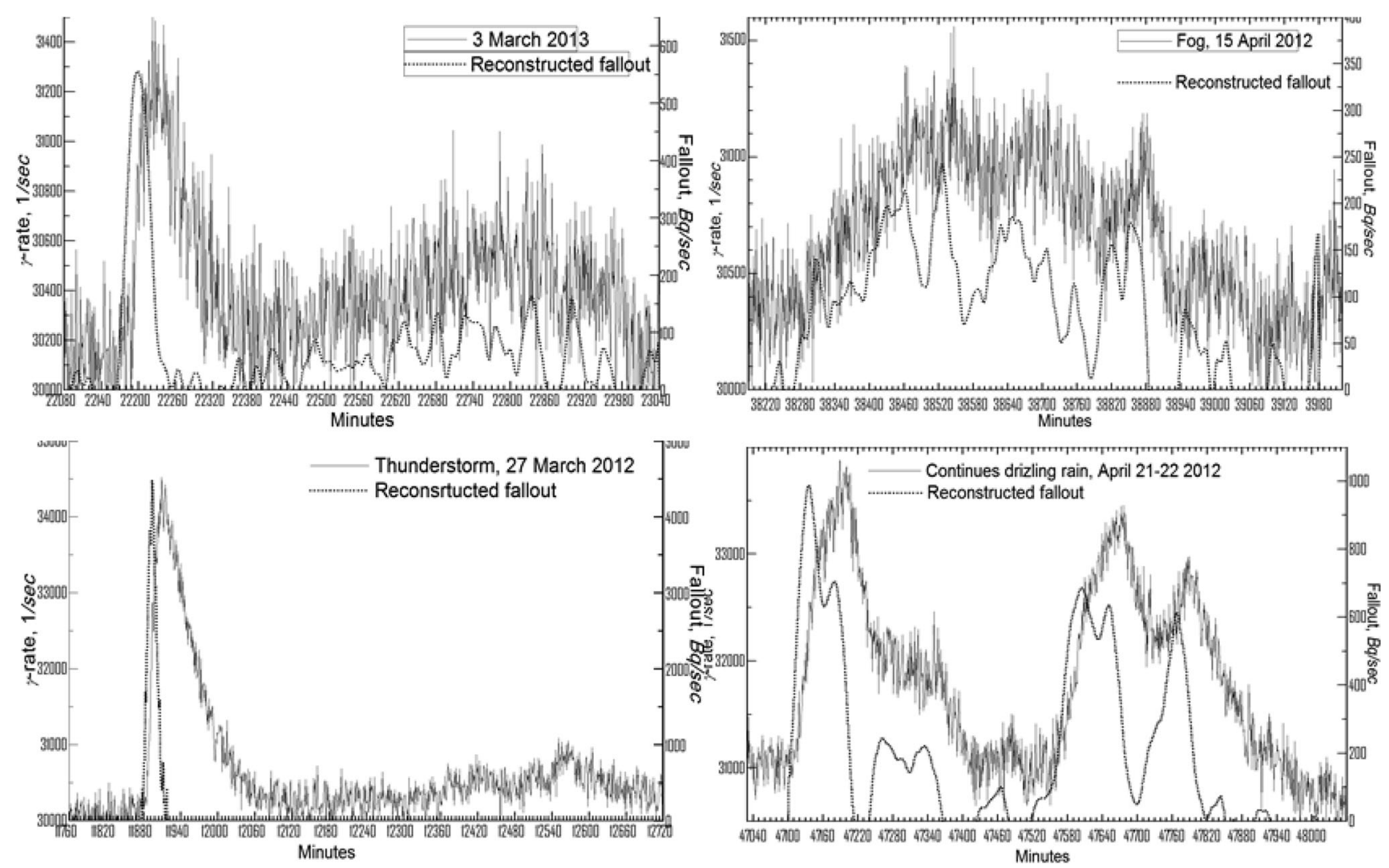

Fig. 3 Examples of simulated fallouts for the precipitation events from Fig. 2

1 min was installed and come into operation in the 2012 (Martin et al. 2014).

Figure 3 also demonstrates corresponding fallout profiles $F(t)$ reconstructed with Eq. 7. Noteworthy variety of profiles, due to differences in parameters and mechanisms caused precipitations. It is very clearly seen in the events of March 3 and 27, 2012 that the leading edge of the gamma enhancement is controlled by fallout rate and the trailing edge after rain termination is determined purely by decay constants.

Figure 4a demonstrates a $\gamma$-rate record, simulated fallout, and specific fallout and rainfall rate for a sequence of two prolonged rains in Jul 23-25 2013 (South America dry season): rain $\mathbf{A}$ lasted from 30 to $55 \mathrm{~h}$ and rain $\mathbf{B}$ from 61 to 83 . The integral characteristics of the events of the rains are listed in Table 1. A dotted curve demonstrates a result of the inverse simulation i.e. that of the $\gamma$-rate from the simulated fallout through Eq. 4.

Comparison of the panels a and b in Fig. 4 demonstrates that the simulated fallout is proportional in the main to the rainfall rate. In the same time, the relation between the specific fallout and rainfall rates is inverse. It is especially well seen for the fallout peaks (marked with 1, 2, 3, 4, 5 in the panel a) all corresponding to low rainfall rate. In the same time several rainfall peaks located between hours 73 and 80 are not accompanied with those of specific fallout. The rest of the rainfall these parameters slightly oscillate near a mean values against significant variation of the rainfall rate especially in the second rain $\mathbf{B}$. One can also see from the Table 1 that at close mean values of $\gamma$-rate and total fallout in the two rains the corresponding rainfall parameters differs by about one order.

The effect of the inverse dependence between the specific fallout and the rainfall is illustrated by Fig. 5 where a dependence of the total accumulated fallout versus the total accumulated rainwater is depicted. Thus the slope of the curve segment is determined by specific fallout rate. A "knee" in the curve at about $8 \mathrm{~mm}$ on the accumulated rainfall axis coincides with the beginning of the rain $\mathbf{B}$ at $3800 \mathrm{~min}$ at time scale of Fig. $4 \mathrm{~b}$. For the rain $\mathbf{A}$ the inclination is 5.1 and for the rain B $0.91 / \mathrm{mm}$.

\section{Discussion}

One can see from Fig. 5 that in spite of the sharp peaks of a few minutes width, the $\gamma$-rate on a larger scale of $\approx 1 \mathrm{~h}$ is linearly proportional to the rainfall rate. A proportionality factor differs in different rains but does not practically change within same rain that means specific fallout 
Minutes
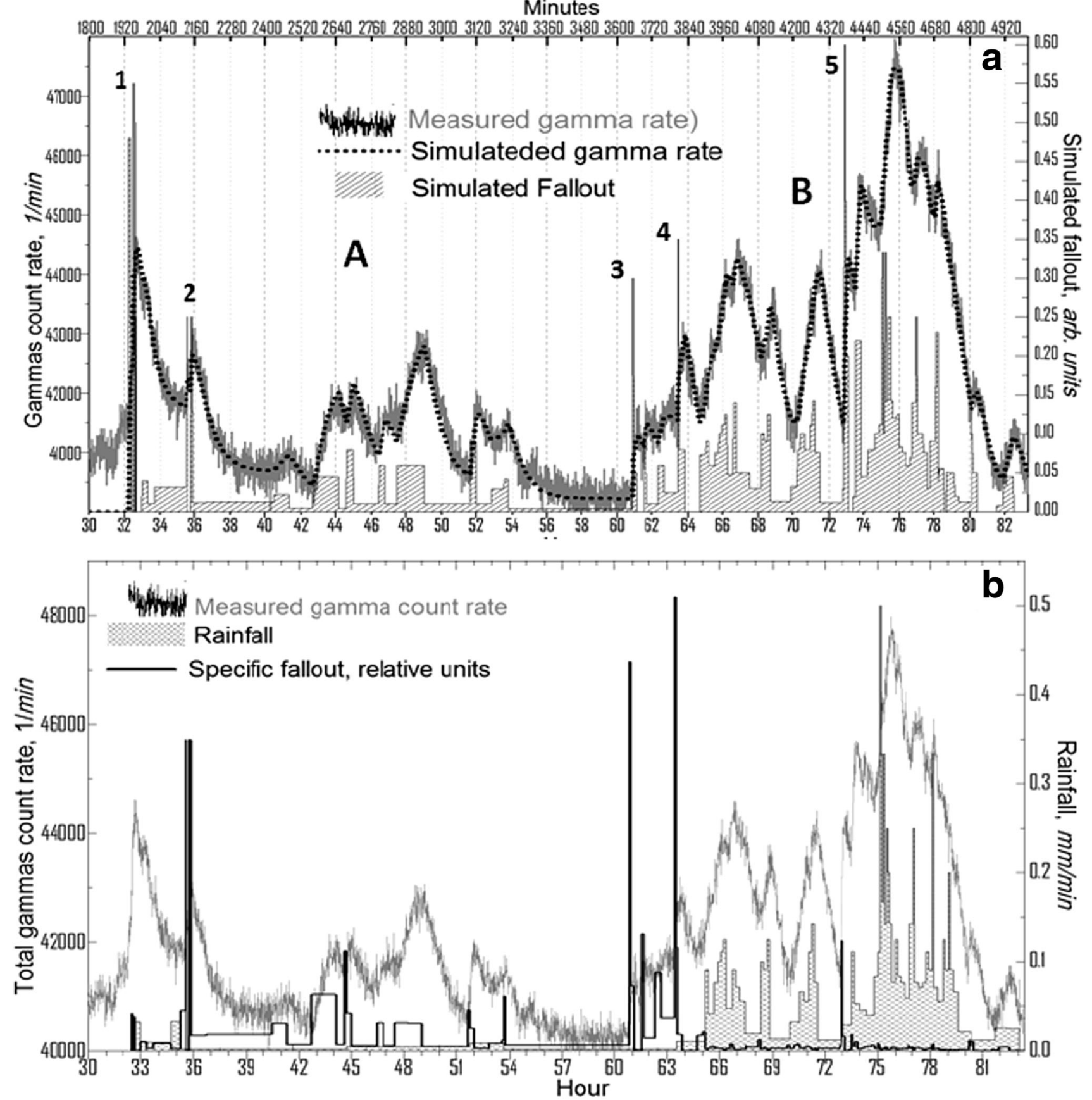

Fig. 4 Comparison of the measured $\gamma$ and rainfall rates with simulated fallout and specific fallout

constancy. The latter feature was also observed by Fujinami (2009). However it does not quite agree with a work of Greenfield et al. (2003) where $\gamma$-rate in same rain is proportional to the (rainfall rate) ${ }^{0.45}$ i.e. specific fallout is proportional to (rainfall rate) ${ }^{-0.55}$. The inverse relation between a rainfall rate and a specific fallout was also observe by Fujinami (2009) and Paatero and Hatakka (1999).
In the same time the integral characteristics of the two rains considered here quite agree with observation of cited authors. The relation obtained by Greenfield et al. (2003) being applied to specific rainfall ratio between separate $\mathbf{B}$ and $\mathbf{A}$ rains results in a value of 0.3 , which is quite close to the observed one of 0.22 . The inverse relation between rainfall and specific fallout was simulated in a numerical model of Minato (2007): according to it the ratio of specific 


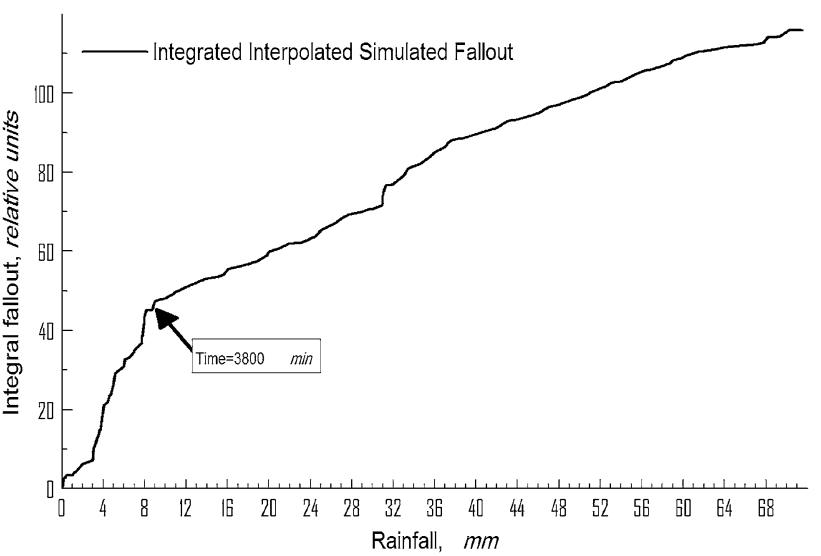

Fig. 5 Dependence of the total accumulated fallout on the total rainfall level for the rainfall shown in Fig. 4

fallouts for rainfall rates 3 and $0.32 \mathrm{~mm} / \mathrm{h}$ is $\approx 0.25$ that is also quite close to the value measured in the present work.

Seems high $\gamma$-rate peaks in Fig. 4 cannot be interpreted the same way as those for the large scales. Probably the effect is not related with precipitations but rather with short rising damps, analogues to that in March 32013 in Fig. 3.

\section{Conclusion}

A numerical method was developed for simulation of the first kind Voltera integral equation with difference kernel, describing variation of nuclide amount produced in the decay chain of ${ }^{214} \mathrm{~Pb}$ and ${ }^{214} \mathrm{Bi}$ with a kernel determined by ratio of precipitating amounts of ${ }^{214} \mathrm{~Pb}$ and ${ }^{214} \mathrm{Bi}$ and their decay half times. The method substitute standard procedure of numerical simulation of the equation via straightforward solution of high order systems of linear equations with an equivalent step-by-step procedure. This is more physically/mathematically transparent and easy adjustable to popular mathematical software.

Application of the method is considered on example of rainfalls in Brazil. Sharp peaks of $\gamma$-radiations coinciding with initial moments of radiation raise were observed in radiation fallout profiles reconstructed with the method developed. The parameters of the observed inverse relation between radioactive fallout and rainfall rates well agree with results of observations and simulations of rainfall related $\gamma$-ray fluxes.

No clear pattern currently exists for a process collection of radiation by raindrops/droplets in the atmosphere/clouds (Papastefanou 2000). The approach developed will be useful in experimental and simulation studies of this phenomenon. In its turn the latter will be a helpful instrument for verification and adjustment of current models of formation of precipitation in clouds and in the atmosphere.
Acknowledgments The authors thank to the Brazilian funding agencies "CAPES (process number PVS-CAPES-ITA 088861/2013)" and " $\mathrm{CNPq}$ " for kindly providing partial financial support to carry out this work

\section{References}

Cardoso LX, Souza SO, Ferreira FCL, Ferreira OC, Barboza E, Alhanati CE (2011) Determination of the specific activityof soiland fertilizers in Sergipe-Brazil, Iternational Journal of Mathematical, Computational, Natural and Physical Engineering vol 5, no: 8, 2011 World Academy of Science, Engineering and Technology Natural and Physical Engineering 5(8)

Evangelista H, Cardoso SNM, Gonçalves AC, Licínio MV, Barboza E, Paschoa AS (2012) Inferring the impact of environmental conditionings over natural gamma-radiation fluxes in a Brazilian tropical site. RadiaT Measurements 47:229-235

Forkapić S, Mrđa D, Vesković M, Todorović N, Bikit K, Nikolov J, Hansmant J (2013) Equilibrium measurement in the air. Rom J Phys 58:S140-S147

Fujinami N (2009) Study of radon progeny distribution and radiation dose rate in the atmosphere. Jpn J Health Phys 44:89-94

Greenfield M, Greenfield A, Domondon T, Tsuchiya S, Tomiyama M (2003) Monitoring precipitation rates using $\gamma$-rays from adsorbed radon progeny as tracers. Jpn J Ap Phys 93:5733-5741

Greenfield M, Ito A, Iwata K, Ishigaki M, Komura K (2008) Determination of rain age via rays from accreted radon progeny. J Ap Phys 104:074912

Horng M, Jiang S (2004) In situ measurements of gamma-ray intensity from radon progeny in rainwater. Rad Meas 38:23-30

Ichiji T, Hattori T (2000) Continuous measurement of environmental gamma radiation in Tokyo using Ge semiconductor detector. http://www.irpa.net/irpa10/cdrom/00611.pdf

Kumar AV, Sitaraman V, Oza RB, Krishnamoorthy TM (1999) Application of a numerical model for the planetary boundary layer to the vertical distribution of radon and its daughter products. Atm Envir 33:4717-4726

Linz P (1985) Analytical and numerical methods for volterra equations S.I.A.M, Philadelphia

Marshall JS, Palmer WM (1948) The distribution of the raindrops with size. J Meteorol 5:165-166

Martin IM, Gomes P, Alves A, Gusev A, Pugacheva G (2014) Monitoring of natural background gamma radiation at ground level in São José Dos Campos, SP, Brazil. Int J Env Ecol Family Urb Stud $\mathrm{p} 4$

Minato S (2007) A simple rainout model for radon daughters. J Nuc Radiochem Sci 8:N1-N3

Nishikawa, Okabe S, Aoki M (1989) Monte carlo calculation of gamma-ray flux density due to atmospheric radon daughters. J Nucl Sci Technol 26:525-529

Nobuyoshi T, Katase A (1982) Rainout-washout model for variation of environmental gamma-ray intensity by precipitation. J Nucl Sci Tech 19:393-409

OECD (1979) Organisation for economic cooperation and development. exposure to radiation from natural radioactivity in building materials. Report by a Group of Experts of the OECD Nuclear Energy Agency, OECD, Paris

Paatero J, Hatakka J (1999) Wet deposition efficiency of short-lived radon-222 progeny in central Finland, Boreal. Env Res 4:285-293

Papastefanou C (2000) Radioactive aerosols. Elsevier, New York

Peters O, Hertlein C, Christensen K (2001) A complexity view of rainfal. Phys Rev Lett 88:01870 
Polyanin AD, Manzhirov AV (2008) Handbook of integral equations, 2nd Edn, Chapman \& Hall/CRC, USA

Press WH, Teukolsky SA, Vetterling WT, Flanner BP (1992) Numerical recipes in Fortran 77: the art of scientific computing 2nd ed, Press Syndicate of the University of Cambridge, Cambridge

Santos IR, Burnett WC, Godoy JM (2008) Radionuclides as tracers of coastal processes in Brazil: review, synthesis, and perspectives, Brazilian. J Oceanogr 56(2)
UNSCEAR (2000) Sources and effects of ionizing radiation. United Nations Scientific Committee on the Radiation, United Nations, New York

Veiga R, Sanches N, Anjos RM, Macario K, Bastos J, Iguatemy M, Aguiarb JG, Santos AMA, Mosquera B, Carvalho C, Filho MB, Umisedo NK (2006) Measurement of natural radioactivity in Brazilian beach sands. Radiat Meas 41:189-196 